\title{
Understanding the effect of cellular structures on mechanical behavior of additively manufactured 316L stainless steel
}

Xin Wang ${ }^{1}$, Baolong Zheng ${ }^{2}$, Kehang $\mathrm{Yu}^{2}$, Sen $\mathrm{Jiang}^{2}$, Yizhang $\mathrm{Zhou}^{2}$, Enrique Lavernia ${ }^{2}$ and Julie Schoenung ${ }^{3}$

${ }^{1}$ University of California, Irvine, IRVINE, California, United States, ${ }^{2}$ University of California, Irvine, United States, ${ }^{3}$ Department of Materials Science and Engineering University of California, Irvine, Irvine, California, United States

Laser engineered net shaping (LENS $\AA$ ), which is one of the most widely used and rapidly developing additive manufacturing (AM) techniques, produces near-net-shaped structures directly from powder or wire feedstock fed into a focused high-power laser beam. It allows manufacturing of materials with complex geometries and compositional gradients that are not easy to achieve with conventional methods. In addition, because of the rapid physical and chemical processes, LENS ${ }^{\circledR}$ produced metals comprise non-equilibrium heterogeneous microstructures that enable attractive strength and ductility combinations in these materials when compared with their conventional counterparts [1,2]. Of particular interest are the fine sub-grain cellular structures widely observed in AM metals [3-6]. To obtain a more complete understanding of the processing-microstructuremechanical property relationships in AM materials, the effect of cellular structures on the mechanical behavior needs to be evaluated.

In the present study, we characterized the microstructure of 316L stainless steel (SS) fabricated via LENS® at multiple scales by combining scanning electron microscopy (SEM), electron backscattered diffraction (EBSD) and scanning transmission electron microscopy (STEM). In situ micro-mechanical testing was performed on 316L SS micro-pillars prepared by focused ion beam (FIB) to investigate the deformation behavior and the effect of cell boundaries on the plasticity of LENS® deposited 316L SS.

Rectangular 316L SS blocks were prepared with a LENS® machine (Optomec Inc., New Mexico, USA) using gas atomized 316L SS powder feedstock inside an Ar atmosphere. The laser power, scan speed and powder feed rate being used are $400 \mathrm{~W}, 17 \mathrm{~mm} / \mathrm{s}$ and $15 \mathrm{~g} / \mathrm{min}$, respectively. Details of the processing conditions and deposition parameters can be found in our previous publications [1,7]. The as-deposited samples are fully dense with a relative density higher than $99 \%$.

Fig. 1 (a) shows an SEM image of the etched surface of an as-deposited 316L SS sample, which reveals a hierarchical microstructure, comprising fine sub-grain cellular structures approximately $1-2 \mu \mathrm{m}$ in size, due to the rapid cooling rate associated with the LENS® processes, and in various orientations. Micro-mechanical testing allows correlation of the cellular structure and the mechanical properties. Fig. 1(b) shows a representative SEM image of an as-milled 316L SS micro-pillar prior to contact with the indenter tip and before in situ compression testing. The microstructure of the micro-pillars after compression was characterized by STEM to explore the deformation mechanisms. As seen in Fig. 2, a high density of dislocations is present in the vicinity of a cell boundary. In addition to a high density of dislocations at the cell boundaries, STEM energy dispersive spectroscopy (EDS) reveals the cell boundaries are decorated by elemental segregation of $\mathrm{Cr}$ and Mo. Our results highlight that the interaction between dislocations and cell boundaries determines the strengthening effect of cell boundaries.

In summary, we will present the application and effectiveness of in situ micro-mechanical testing and electron microscopy to investigate the microstructure-mechanical property relationships in AM fabricated metallic materials with non-equilibrium hierarchical microstructures. In the case of LENS@ deposited 316L SS, the characteristic sub-grain cellular structures play an important role in determining the strength and anisotropy of 
the material via dislocation-cell boundary interactions. Our results provide insights into the opportunity to apply cell boundary engineering in AM for enhanced mechanical properties.
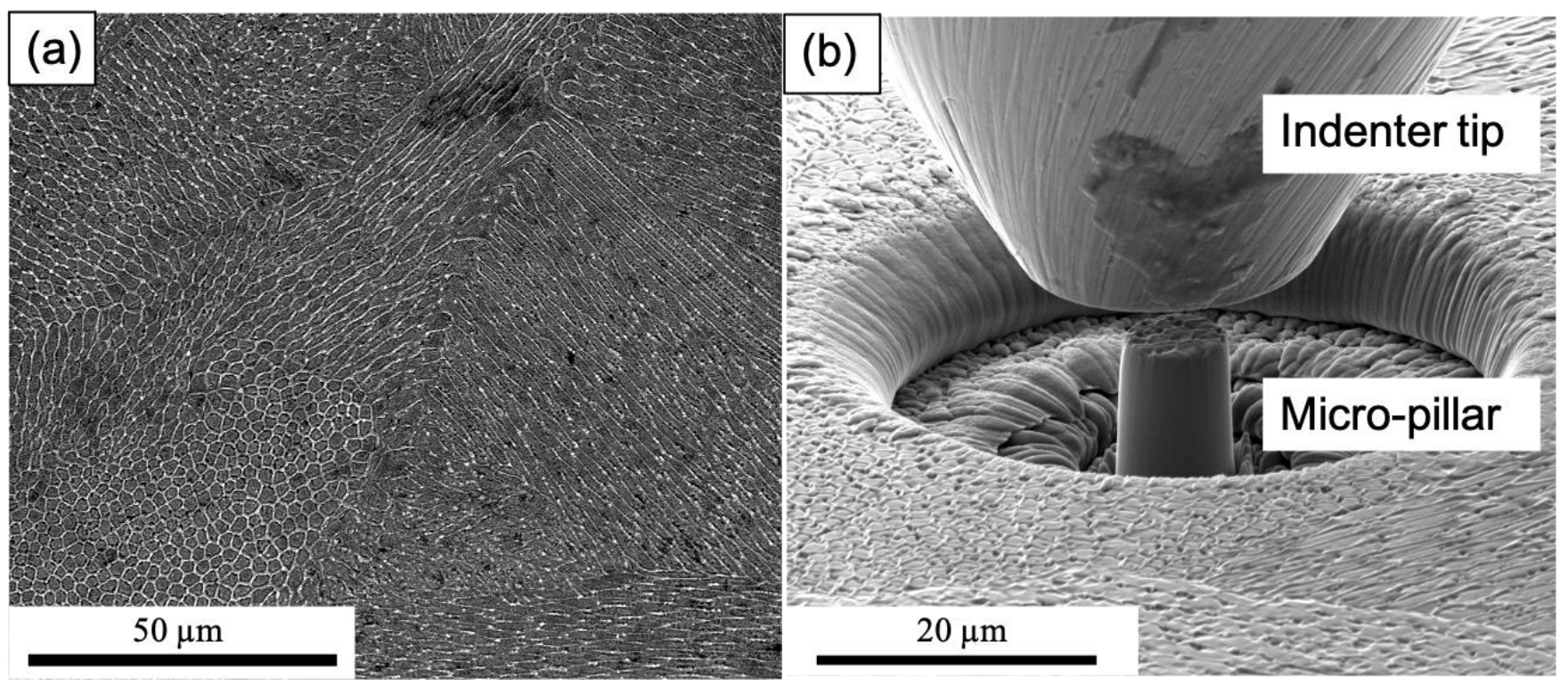

Figure 1. Figure 1 (a) SEM micrograph of the etched surface of an as-deposited 316L SS. (b) Representative SEM image of an as-milled 316L SS micro-pillar.

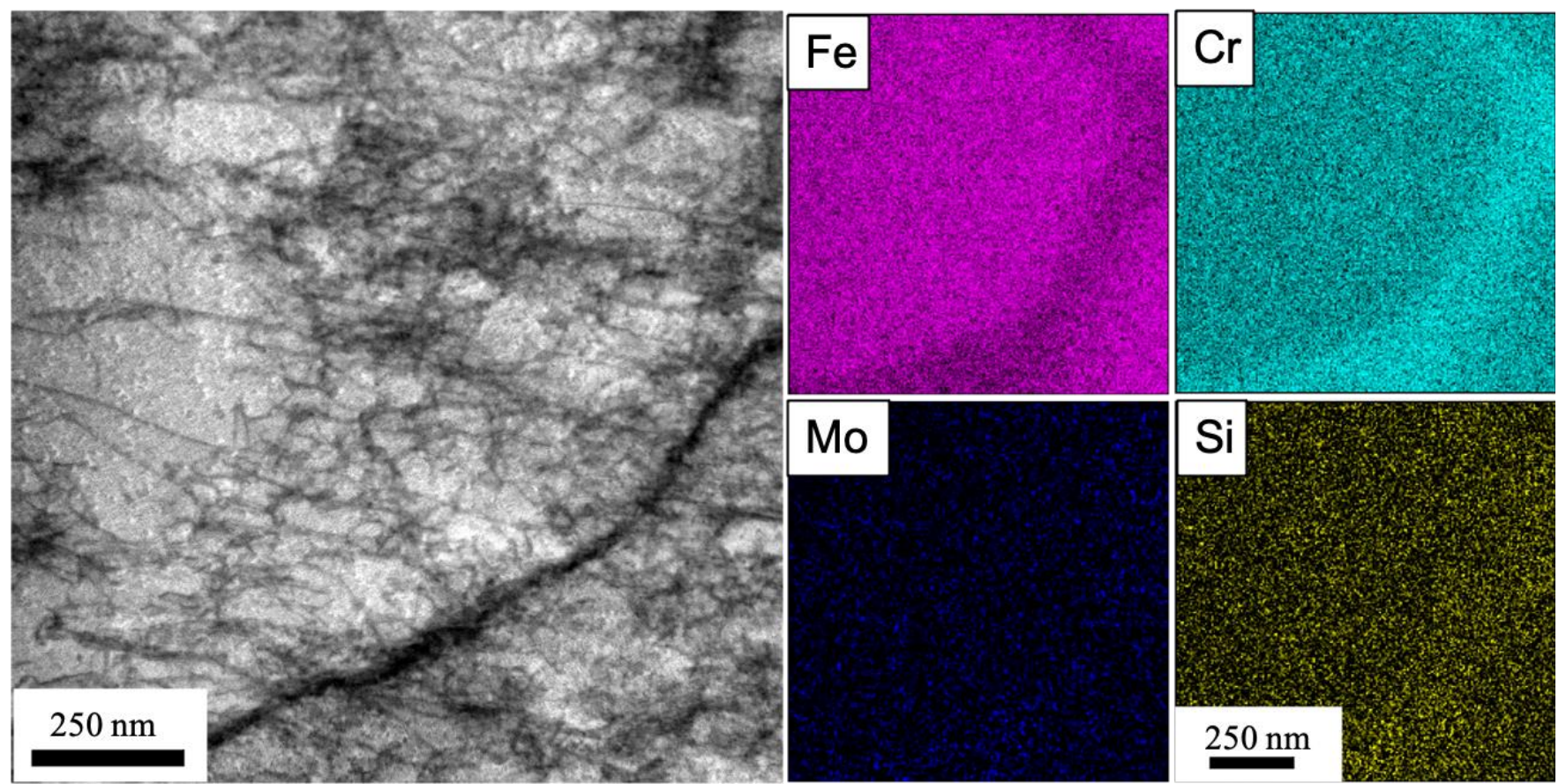

Figure 2. Figure 2 Post-mortem STEM bright-field image and EDS showing the cellular structure after in situ compression testing.

\section{References}

[1] B. Zheng, J.C. Haley, N. Yang, J. Yee, K.W. Terrassa, Y. Zhou, E.J. Lavernia, J.M. Schoenung, Materials Science and Engineering: A 764 (2019) 138243.

[2] T.R. Smith, J.D. Sugar, C. San Marchi, J.M. Schoenung, Acta Materialia 164 (2019) 728-740. 
[3] Y.M. Wang, T. Voisin, J.T. McKeown, J. Ye, N.P. Calta, Z. Li, Z. Zeng, Y. Zhang, W. Chen, T.T. Roehling, R.T. Ott, M.K. Santala, P.J. Depond, M.J. Matthews, A.V. Hamza, T. Zhu, Nature Materials 17 (2018) 63-71. [4] Z. Li, B. He, Q. Guo, Scripta Materialia 177 (2020) 17-21.

[5] Q. Han, Y. Gu, R. Setchi, F. Lacan, R. Johnston, S.L. Evans, S. Yang, Additive Manufacturing 30 (2019) 100919.

[6] L. Thijs, K. Kempen, J.-P. Kruth, J. Van Humbeeck, Acta Materialia 61 (2013) 1809-1819.

[7] K.L. Terrassa, T.R. Smith, S. Jiang, J.D. Sugar, J.M. Schoenung, Materials Science and Engineering: A 765 (2019) 138269. 